University of Wollongong

Research Online

Faculty of Informatics - Papers (Archive)

Faculty of Engineering and Information

Sciences

January 2001

\title{
Values of Minors of an Infinite Family of D-Optimal Designs and Their Application to the Growth Problem
}

C. Koukouvinos

National Technical University of Athens, Greece

M. Mitrouli

University of Athens, Greece

Jennifer Seberry

University of Wollongong, jennie@uow.edu.au

Follow this and additional works at: https://ro.uow.edu.au/infopapers

Part of the Physical Sciences and Mathematics Commons

\section{Recommended Citation}

Koukouvinos, C.; Mitrouli, M.; and Seberry, Jennifer: Values of Minors of an Infinite Family of D-Optimal Designs and Their Application to the Growth Problem 2001.

https://ro.uow.edu.au/infopapers/321

Research Online is the open access institutional repository for the University of Wollongong. For further information contact the UOW Library: research-pubs@uow.edu.au 


\title{
Values of Minors of an Infinite Family of D-Optimal Designs and Their Application to the Growth Problem
}

\author{
Abstract \\ We obtain explicit formulae for the values of the $2 v-j$ minors, $j=0,1,2$ of D-optimal designs of order $2 v$ \\ $=\mathrm{x} 2+\mathrm{y} 2$, v odd, where the design is constructed using two circulant or type 1 incidence matrices of either \\ two $\operatorname{SBIBD}(2 s 2+2 s+1, s 2, s 2-s / 2)$ or $2-\{2 s 2+2 s+1 ; s 2, s 2 ; s(s-1)\}$ sds. This allows us to obtain \\ information on the growth problem for families of matrices with moderate growth. Some of our \\ theoretical formulae imply growth greater than $2(2 s 2+2 s+1)$ but experimentation has not yet supported \\ this result. An open problem remains to establish whether the $(1,-1) \mathrm{CP}$ incidence matrices of certain \\ SBIBDs and $2-\{2 s 2+2 s+1 ; s 2, s 2 ; s(s-1)\}$ sds which yield D-optimal designs, can have growth \\ greater than $2 \mathrm{v}$.

\section{Keywords} \\ D-optimal designs, SBIBD, supplementary difference sets, Gaussian elimination, growth, complete \\ pivoting, AMS Subject Classification: 05B20, 15A15, 65F05, $65 \mathrm{G} 05$.

\section{Disciplines} \\ Physical Sciences and Mathematics

\section{Publication Details} \\ This article was originally published as: Koukouvinos, C, Mitrouli, M \& Seberry, J, Values of Minors of an \\ Infinite Family of D-Optimal Designs and Their Application to the Growth Problem, SIAM Journal on Matrix \\ Analysis and Applications, 2001, 23(1), 1-14. Copyright 2001 Society for Industrial and Applied \\ Mathematics (SIAM).
}




\title{
Values of Minors of an Infinite Family of $D$-Optimal Designs and Their Application to the Growth Problem
}

\author{
C. Koukouvinos, M. Mitrouli†and Jennifer Seberry ${ }^{\ddagger}$
}

December 1, 2000

\begin{abstract}
We obtain explicit formulae for the values of the $2 v-j$ minors, $j=0,1,2$ of $D$-optimal designs of order $2 v=x^{2}+y^{2}, v$ odd, where the design is constructed using two circulant or type 1 incidence matrices of either two $\operatorname{SBIBD}\left(2 s^{2}+2 s+1, s^{2}, \frac{s^{2}-s}{2}\right)$ or $2-\left\{2 s^{2}+2 s+\right.$ $\left.1 ; s^{2}, s^{2} ; s(s-1)\right\}$ sds. This allows us to obtain information on the growth problem for families of matrices with moderate growth. Some of our theoretical formulae imply growth greater than $2\left(2 s^{2}+2 s+1\right)$ but experimentation has not yet supported this result. An open problem remains to establish whether the $(1,-1)$ CP incidence matrices of certain SBIBDs and $2-\left\{2 s^{2}+2 s+1 ; s^{2}, s^{2} ; s(s-1)\right\}$ sds which yield $D$-optimal designs, can have growth greater than $2 v$.
\end{abstract}

Key Words and Phrases: D-optimal designs, SBIBD, supplementary difference sets, Gaussian elimination, growth, complete pivoting.

AMS Subject Classification: 05B20, 15A15, 65F05, 65G05.

\section{Introduction}

A $D$-optimal design of order $n$ is an $n \times n$ matrix with entries \pm 1 having maximum determinant. In the present paper we evaluate the $2 v-j, j=0,1,2$ minors for $(1,-1)$ incidence matrices of certain SBIBDs which yield $D$-optimal designs. For the purpose of this paper we will define a $\operatorname{SBIBD}(v, k, \lambda)$ to be a $v \times v$ matrix, $\mathrm{B}$, with entries 0 or 1 , which has exactly $k$ entries +1 and $v-k$ entries 0 in each row and column and for which the inner product of any distinct pairs of rows and columns is $\lambda$. The $(1,-1)$ incidence matrix of $B$ is obtained by letting $A=2 B-J$, where $J$ is the $v \times v$ matrix with entries all +1 . We write $I$ for the identity matrix of order $v$. Then we have

$$
B B^{T}=(k-\lambda) I+\lambda J
$$

and

$$
A A^{T}=4(k-\lambda) I+(v-4(k-\lambda)) J
$$

\footnotetext{
${ }^{*}$ Department of Mathematics, National Technical University of Athens, Zografou 15773, Athens, Greece.

${ }^{\dagger}$ Department of Mathematics, University of Athens, Panepistemiopolis 15784, Athens, Greece.

${ }^{\ddagger}$ School of Information Technology and Computer Science, University of Wollongong, Wollongong, NSW, 2522, Australia.
} 
It can be easily shown that

$$
\operatorname{det} B=(k-\lambda)^{\frac{v-1}{2}} \sqrt{k+(v-1) \lambda}
$$

and since $\lambda(v-1)=k^{2}-k$

$$
\operatorname{det} A=2^{v-1}(k-\lambda)^{\frac{v-1}{2}}|v-2 k|
$$

In this paper we also study the application of the computed values of the minors to the growth problem for $S B I B D\left(2 s^{2}+2 s+1, s^{2}, \frac{1}{2} s(s-1)\right)$, which is Brouwer's design and which yields a $D$-optimal design.

Let $A=\left[a_{i j}\right] \in \mathcal{R}^{n \times n}$. We reduce $A$ to upper triangular form by using Gaussian elimination with complete pivoting (GECP) [19]. Let $A^{(k)}=\left[a_{i j}^{(k)}\right]$ denote the matrix obtained after the first $k$ pivoting operations, so $A^{(n-1)}$ is the final upper triangular matrix. A diagonal entry of that final matrix will be called a pivot. Matrices with the property that no exchanges are actually needed during GECP are called completely pivoted (CP). Let $g(n, A)=\max _{i, j, k}\left|a_{i j}^{(k)}\right| /\left|a_{11}^{(0)}\right|$ denote the growth associated with GECP on $A$ and $g(n)=\sup \left\{g(n, A) / A \in \mathcal{R}^{n \times n}\right\}$. The problem of determining $g(n)$ for various values of $n$ is called the growth problem.

The determination of $g(n)$ remains one of the major unsolved problems in numerical analysis. See [9] for a detailed description of the problem. One of the curious frustrations of the growth problem is that it is quite difficult to construct any examples of $n \times n$ matrices $A$ other than Hadamard matrices for which $g(n, A)$ is even close to $n$. The equality $g(n, A)=n$ has been proved for a certain class of $n \times n$ Hadamard matrices [4]. It has also been observed that weighing matrices of order $n$ can give $g(n, A)=n-1$ [12]. In [11] the pivot structure of $(1,-1)$ incidence matrices of $S B I B D(v, k, \lambda)$ is studied. In the present paper we get values for the pivots of $2-\left\{2 s^{2}+2 s+1 ; s^{2}, s^{2} ; s(s-1)\right\}$ sds, and $D$-optimal designs made from them. Calculations have given moderate values of growth for $D$-optimal designs. An open problem concerning the possibility of finding $(1,-1) 2 v \times 2 v \mathrm{CP} D$-optimal designs having growth greater than $2 v$ is posed.

Notation 1. Write $A$ for a matrix of order $n$ whose initial pivots are derived from matrices with CP structure. Write $A(j)$ for the absolute value of the determinant of the $j \times j$ principal submatrix in the upper lefthand corner of the matrix $A$ and $A[j]$ for the absolute value of the determinant of the $(n-j) \times(n-j)$ principal submatrix in the bottom righthand corner of the matrix $A$. Throughout this paper when we have used $i$ pivots we then find all possible values of the $A(n-i)$ minors. Hence, if any minor is CP it must have one of these values. The magnitude of the pivots appearing after the application of $\mathrm{GE}$ operations on a $\mathrm{CP}$ matrix $\mathrm{W}$ is given by

$$
p_{j}=W(j) / W(j-1), \quad j=1,2, \ldots, n, \quad W(0)=1 .
$$

In particular for a $\mathrm{CP} \operatorname{SBIBD}(v, k, \lambda), A$,

$$
p_{v}=A(v) / A(v-1), \quad p_{v-1}=A(v-1) / A(v-2) .
$$

We use the notation $M_{j}$ to denote the $j \times j$ minor of $A$.

For completeness we give the determinant simplification theorem in Appendix as we use it extensively in this paper. 


\section{$2 \quad D$-optimal designs of order $2 v \equiv 2(\bmod 4)$ from $S B I B D s$}

Let $d_{n}$ denote the maximum determinant of all $n \times n$ matrices with elements \pm 1 . It follows from Hadamard's inequality that $d_{n} \leq n^{\frac{n}{2}}$ and it is easily shown that equality can only hold if $n=1$ or 2 or if $n \equiv 0(\bmod 4)$. We shall here be concerned with the case $n \equiv 2(\bmod 4), n \neq 2$. Ehlich [6], showed that

$$
d_{n} \leq(2 n-2)(n-2)^{\frac{n}{2}-1}
$$

and equality can hold only if $2 n-2=x^{2}+y^{2}$, where $x$ and $y$ are integers.

Recently two infinite series of $n \times n(n \equiv 2(\bmod 4))$ matrices with elements \pm 1 and maximum determinant were discovered. The first series (Koukouvinos-Kounias-Seberry-Singer or KKSS) [10], exists for $n=2\left(q^{2}+q+1\right)$ where $q$ is a prime power. The second series ( Whiteman-Brouwer or $W B)$ [18], exists for $n=2\left(2 q^{2}+2 q+1\right)$ where $q$ is an odd prime power.

For the purpose of this paper we will define two supplementary difference sets $2-\left\{v ; k_{1}, k_{2} ; \lambda\right\}$, abbreviated as sds, to be two circulant (or type 1) $v \times v$ matrices $B_{1}$ and $B_{2}$, with entries 0 or 1 , which have exactly $k_{i}$ entries +1 and $v-k_{i}$ entries $0, i=1,2$ respectively, in each row and column and for which the inner product of any pair of rows is $\lambda$. The $(1,-1)$ incidence matrices of $B_{i}$, are obtained by letting $A_{i}=2 B_{i}-J, \quad i=1,2$.

The family of $S B I B D\left(2 s^{2}+2 s+1, s^{2}, \frac{1}{2} s(s-1)\right.$, for $s$ an odd prime power, has been found by Brouwer [3]. For $s=2$, the $\operatorname{SBIBD}(13,4,1)$ comes from the projective plane. The case for $s=4$, the $\operatorname{SBIBD}(41,16,6)$ is given by Bridges, Hall and Hayden [2] and independently by van Trung [16]. The case for $s=6$, the $\operatorname{SBIBD}(85,36,15)$ is given as unknown by van Trung [17, p.84] and Beth, Jungnickel and Lenz [1, p.625]. However, for $s=6$ Gysin [8] gives the first $2-\{85 ; 36,36 ; 30\}$ sds. For $s=8$ Djokovic [5] gives the first $2-\{145 ; 64,64 ; 56\}$ sds. Georgiou and Koukouvinos [7] give further results for $s=6$ and $s=8$. In addition, for $s=3$, i.e. $2-\{25 ; 9,9 ; 6\}$, there is a type 1 solution in the group $Z_{5} \times Z_{5}$.

These $2-\left\{2 s^{2}+2 s+1 ; s^{2}, s^{2} ; s(s-1)\right\}$ sds have $(1,-1)$ incidence matrices which satisfy

$$
A_{1} A_{1}^{T}+A_{2} A_{2}^{T}=\left(4 s^{2}+4 s\right) I+2 J
$$

Let $R$ and $S$ be permutation matrices of order $v$. Then $A$ given by

$$
\left[\begin{array}{cc}
P & P \\
R P S & -R P S
\end{array}\right] \text { or }\left[\begin{array}{cc}
A_{1} & A_{2} \\
A_{2}^{T} & -A_{1}^{T}
\end{array}\right]
$$

are $D$-optimal designs of order $2 v \equiv 2(\bmod 4)$ of the WB family. We can say the WB family is constructed from $2-\left\{2 s^{2}+2 s+1 ; s^{2}, s^{2} ; s(s-1)\right\}$ sds. Note $A_{1}=A_{2}$ for the WB family.

We can write

$$
A A^{T}=(2 v-2) I_{2 v}+2 I_{2} \times J_{v} .
$$

It is easy to use the determinant simplification theorem to see that

$$
\operatorname{det} A=2^{v}(2 v-1)(v-1)^{v-1}
$$


Since $A$ has been constructed using the $2-\left\{2 s^{2}+2 s+1 ; s^{2}, s^{2} ; s(s-1)\right\}$ sds,

$$
\operatorname{det} A=M_{2 v}=2^{(2 s+1)^{2}}(2 s+1)^{2}(s(s+1))^{2 s(s+1)} .
$$

\subsection{Minors of Size $(2 v-1)$}

To find the $(2 v-1) \times(2 v-1)$ minors we remove the first row and column of $A$ to get $B$. Now, $\operatorname{det} A A^{T}=\operatorname{det} \Delta(h, i, j, k, m)$ where

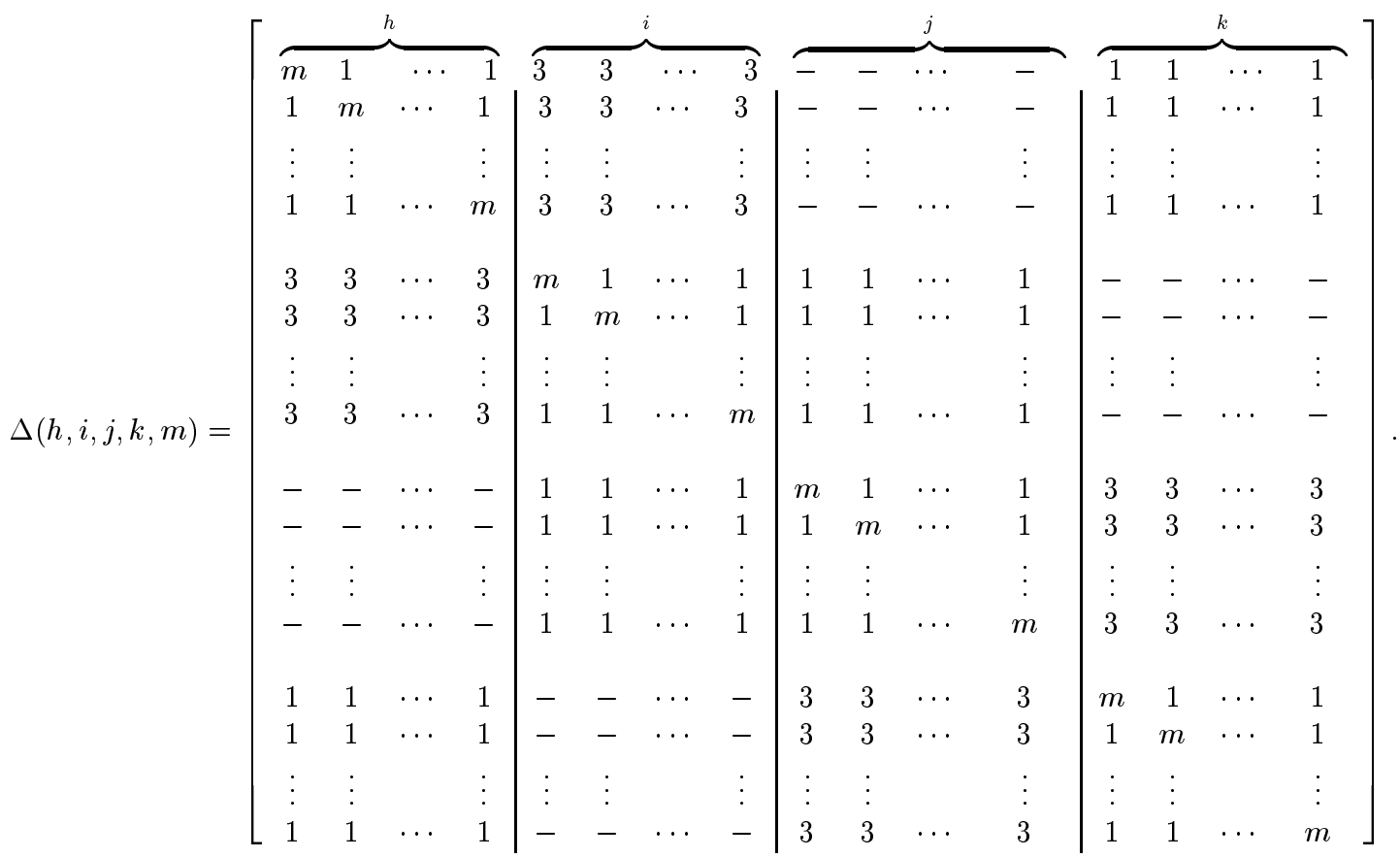

$m=h+i+j+k$. Then by the determinant simplification theorem

$$
\operatorname{det} \Delta(h, i, j, k, m)=(m-1)^{m-4}\left|\begin{array}{cccc}
m-1+h & 3 h & -h & h \\
3 i & m-1+i & i & -i \\
-j & j & m-1+j & 3 j \\
k & -k & 3 k & m-1+k
\end{array}\right|
$$

and det $\Delta(h, i, j, k, m)=(m-1)^{4}+(m-1)^{3}(i+j+h+k)-8(m-1)^{2}(j k+i h)-16(m-$ 1) $(j k(i+h)+i h(j+k))$.

Now $\operatorname{det} B B^{T}$ is obtained from $\Delta(h, i, j, k, m)$ by removing a row and the corresponding column. Thus det $B B^{T}$ is $\Delta(h-1, i, j, k, m-1)$ or $\Delta(h, i-1, j, k, m-1)$ or $\Delta(h, i, j-1, k, m-1)$ or $\Delta(h, i, j, k-1, m-1)$.

Lemma 1 The $(2 v-1) \times(2 v-1)$ minors of the D-optimal designs of the $W B$ series are

$$
M_{2 v-1}=2^{4 s(s+1)}(2 s+1) s^{2 s^{2}+2 s-1}(s+1)^{2 s^{2}+2 s} \text { and } 2^{4 s(s+1)}(2 s+1) s^{2 s^{2}+2 s}(s+1)^{2 s^{2}+2 s-1}
$$

where $s$ is an odd prime power, $s=2,4,6$ or 8 . 
Proof. Here we use the $(1,-1)$ incidence matrices of the $2-\left\{2 s^{2}+2 s+1 ; s^{2}, s^{2} ; s(s-1)\right\}$ sds. By the reasoning above, with $v=2 s^{2}+2 s+1, h=j=s^{2}, i=k=s^{2}+2 s+1, m=4 s^{2}+4 s+2$, substituted into $\Delta(h-1, i, j, k, m-1)=\Delta(h, i, j-1, k, m-1)$, we obtain the result. Specifically the determinant is the square root of the determinant given by

$$
\begin{aligned}
& \left(4 s^{2}+4 s\right)^{4 s^{2}+4 s-3}\left|\begin{array}{cccc}
5 s^{2}+4 s-1 & 3 s^{2}-3 & -s^{2}+1 & s^{2}-1 \\
3 s^{2}+6 s+3 & 5 s^{2}+6 s+1 & s^{2}+2 s+1 & -s^{2}-2 s-1 \\
-s^{2} & s^{2} & 5 s^{2}+4 s & 3 s^{2} \\
s^{2}+2 s+1 & -s^{2}-2 s-1 & 3 s^{2}+6 s+3 & 5 s^{2}+6 s+1
\end{array}\right| \\
& =2^{2\left(4 s^{2}+4 s\right)}\left(s^{2}+s\right)^{4 s^{2}+4 s-2}(s+1)^{2}(2 s+1)^{2}=\left[2^{4 s^{2}+4 s} s^{2 s^{2}+2 s-1}(s+1)^{2 s^{2}+2 s}(2 s+1)\right]^{2} \\
& \text { det } \Delta(h, i-1, j, k, m-1)=\operatorname{det} \Delta(h, i, j, k-1, m-1)=\left[2^{4 s^{2}+4 s} s^{2 s^{2}+2 s}(s+1)^{2 s^{2}+2 s-1}(2 s+1)\right]^{2} \\
& \text { gives the second result. }
\end{aligned}
$$

\subsection{Minors of size $(2 v-2)$}

As the partitioned matrix $A$ of the $D$-optimal design is composed from $2-\left\{v ; k_{1}, k_{2} ; \lambda\right\}$ supplementary difference sets, these are in fact $2-\left\{2 s^{2}+2 s+1 ; s^{2}, s^{2} ; s^{2}-s\right\}$ sds. We will use $k=k_{1}=k_{2}$ for all our calculations. Using the formula for the inner product of the rows of the $(1,-1)$ incidence matrix formed from these sds we see that the inner product is $2 v-4\left(k_{1}+k_{2}-\lambda\right)=2$.

We now return to $A$ with two rows and columns removed to find the generic matrix. We have not included this in expanded form except for one case but moved straight to the determinant after it has been simplified using the Determinant Simplification Theorem, of the matrix $D$ given by

$$
\left[\begin{array}{cccccccc}
2 v-2 & 2 u_{2} & 2 u_{3} & 4 u_{4} & -2 u_{5} & 0 & 0 & 2 u_{8} \\
2 u_{1} & 2 v-2 & 4 u_{3} & 2 u_{4} & 0 & -2 u_{6} & 2 u_{7} & 0 \\
2 u_{1} & 4 u_{2} & 2 v-2 & 2 u_{4} & 0 & 2 u_{6} & -2 u_{7} & 0 \\
4 u_{1} & 2 u_{2} & 2 u_{3} & 2 v-2 & 2 u_{5} & 0 & 0 & -2 u_{8} \\
-2 u_{1} & 0 & 0 & 2 u_{4} & 2 v-2 & 2 u_{6} & 2 u_{7} & 4 u_{8} \\
0 & -2 u_{2} & 2 u_{3} & 0 & 2 u_{5} & 2 v-2 & 4 u_{7} & 2 u_{8} \\
0 & 2 u_{2} & -2 u_{3} & 0 & 2 u_{5} & 4 u_{6} & 2 v-2 & 2 u_{8} \\
2 u_{1} & 0 & 0 & -2 u_{4} & 4 u_{5} & 2 u_{6} & 2 u_{7} & 2 v-2
\end{array}\right] .
$$

This gives the determinant of $A$ with two rows and columns removed, as $\left(4 s^{2}+4 s\right)^{2 s^{2}+2 s-4} \sqrt{\operatorname{det} D}$.

To calculate the minors of size $(2 v-2)$ we distinguish two major Cases: Case I where the two rows removed to form the minor came from the same part of the $D$-optimal design that is they have inner product 2; Case II where the two rows removed to form the minor came from different parts of the $D$-optimal design that is they have inner product zero. This leads to the following four subcases: 
Case Ia $\left[\begin{array}{ll}x & y \\ x & \bar{y}\end{array}\right]$ where the $(1,1)$ and $(2,1)$ elements have the same sign, the $(1,2)$ element and the $(2,2)$ element have opposite signs, and the inner product of row one and two with each other is 2 . The inner product of the first two rows with the next $(v-i)$ rows is $+i$ and the inner product of row one and two with the $v+3-i$ to $2 v$ th rows is $2-i$, where $i=2$ or 0 .

Case Ib $\left[\begin{array}{ll}x & y \\ x & \bar{y}\end{array}\right]$ where the $(1,1)$ and $(2,1)$ elements have the same sign, the $(1,2)$ element and the $(2,2)$ element have opposite signs, and the inner product of rows one and two with each other is +2 . The inner product of rows one and two with next $v-i$ rows is $+i$ and the inner product of the first two rows with rows $v+3-i$ to $2 v$ is $2-i$, where $i=2$ or 0 .

Case IIa $\left[\begin{array}{ll}x & y \\ x & \bar{y}\end{array}\right]$ where the $(1,1)$ element and the $(2,1)$ element have the same signs, the $(1,2)$ element and the $(2,2)$ element have different signs, and the inner product of rows one and two with each other is zero. Rows 3 to $v+1$ have inner product +2 with row one and zero with row two. Rows $v+2$ to $2 v$ have inner product zero with row one and +2 with row two.

Case IIb $\left[\begin{array}{ll}x & y \\ x & y\end{array}\right]$ where the $(1,1)$ element and the $(2,1)$ element have the same signs, the $(1,2)$ element and the $(2,2)$ element also have the same sign , and the inner product of row one and two with each other is zero. Rows 3 to $v+1$ have inner product +2 with row one and zero with row two. Rows $v+2$ to $2 v$ have inner product zero with row one and +2 with row two.

A careful study of cases leads to only the cases now listed as Case III not being permutation equivalent to one of Cases I and II.

Case III $\left[\begin{array}{ll}x & y \\ x & \bar{y}\end{array}\right]$ where one of the columns in the submatrix has two identical elements and the other has two different elements. The inner product of rows one and two with each other is zero. Each of row one and row two have inner product $i$ with $v-1$ other rows and $2-i$ with the remainder of the rows, $i=2$ or 0 .

\section{Case Ia}

We have the possible $2 \times 2$ submatrices:

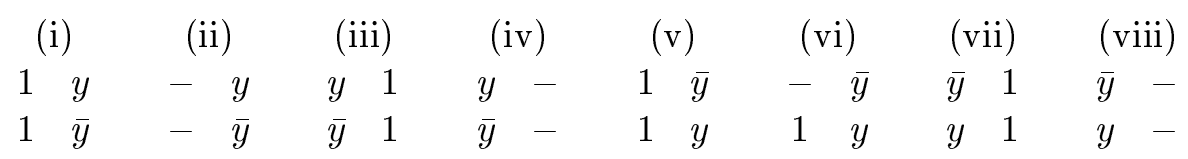

Since permutation of columns 1 and 2 has no effect on $M_{2 v-2}$ cases (i) and (iii), (v) and (vii), (ii) and (iv), and (vi) and (viii) give the same values. Cases (i) and (v) give the same values depending on whether $y=1$ or -1 and similarly for cases (ii) and (vi). This leaves the following submatrices for Case Ia: 
(i)

(ii)

$1 y-y$

$1 \bar{y}-\bar{y}$

However, in Cases I rows 1 and 2 may be permuted without altering the value of $M_{2 v-2}$, so, without loss of generality we may consider $y=1$. Also, without loss of generality, we may permute rows three to $2 v$ of the matrix so rows three to $v$ have inner product +2 with rows one and two. The inner product of rows one and two with the next $v-2$ rows is 2 , and the inner product of rows 1 and 2 with rows $v+1$ to $2 v$ is zero. This yields the following cases for Case Ia:

\begin{tabular}{|c|c|c|c|c|c|c|c|c|c|}
\hline \multirow{2}{*}{\multicolumn{2}{|c|}{$\begin{array}{c}2 \times 2 \\
\text { submatrix }\end{array}$}} & \multicolumn{8}{|c|}{ Number of Rows of Each Type Ia } \\
\hline & & $u_{1}$ & $u_{2}$ & $u_{3}$ & $u_{4}$ & $u_{5}$ & $u_{6}$ & $u_{7}$ & $u_{8}$ \\
\hline $\begin{array}{l}1 \\
1\end{array}$ & 1 & $\lambda-1$ & $k-\lambda-1$ & $k-\lambda$ & $v-2 k+\lambda$ & $\lambda$ & $k-\lambda$ & $k-\lambda$ & $v-2 k+\lambda$ \\
\hline - & $\begin{array}{l}1 \\
-\end{array}$ & $\lambda$ & $k-\lambda$ & $k-\lambda-1$ & $v-2 k+\lambda-1$ & $\lambda$ & $k-\lambda$ & $k-\lambda$ & $v-2 k+\lambda$ \\
\hline
\end{tabular}

To illustrate the derivation of the tables such as Table 1 we give Case Ia as an example.

\begin{tabular}{ccc|ccc|l}
1 & $y$ & & - & $y$ & & Inner Product \\
1 & $\bar{y}$ & & - & $\bar{y}$ & & \\
\hline 1 & 1 & & 1 & 1 & & \\
$\vdots$ & $\vdots$ & $\lambda-1$ & $\vdots$ & $\vdots$ & $\lambda$ & \\
1 & 1 & & 1 & 1 & & $v-2$ rows is 2 \\
1 & - & & 1 & - & & \\
$\vdots$ & $\vdots$ & $k-\lambda-1$ & $\vdots$ & $\vdots$ & $k-\lambda$ & which have \\
1 & - & & 1 & - & & inner \\
- & 1 & & - & 1 & & product 2 \\
$\vdots$ & $\vdots$ & $k-\lambda$ & $\vdots$ & $\vdots$ & $k-\lambda-1$ & with rows \\
- & 1 & & - & 1 & & one and two \\
- & - & & - & - & & \\
$\vdots$ & $\vdots$ & $v-2 k+\lambda$ & $\vdots$ & $\vdots$ & $v-2 k+\lambda-1$ & \\
- & - & & - & - & & \\
\hline 1 & 1 & & 1 & 1 & & \\
$\vdots$ & $\vdots$ & $\lambda$ & $\vdots$ & $\vdots$ & $\lambda$ & \\
1 & 1 & & 1 & 1 & & \\
1 & - & & 1 & - & & $v$ rows \\
$\vdots$ & $\vdots$ & $k-\lambda$ & $\vdots$ & $\vdots$ & $k-\lambda$ & which have \\
1 & - & & 1 & - & & inner \\
- & 1 & & - & 1 & & product 0 \\
$\vdots$ & $\vdots$ & $k-\lambda$ & $\vdots$ & $\vdots$ & $k-\lambda$ & with rows \\
- & 1 & & - & 1 & & one and two \\
- & - & & - & - & & \\
$\vdots$ & $\vdots$ & $v-2 k+\lambda$ & $\vdots$ & $\vdots$ & $v-2 k+\lambda$ & \\
- & - & & - & - & &
\end{tabular}

\section{Case Ib}

A similar argument to that for Case Ia shows that using the permutations of columns 1 and 2 we only have to consider the submatrices 
(i)

(ii)

$1 \quad y-y$

$1 y-y$

for $y=1$ and $y=-1$. We make the inner product with rows three to $v$ with the first two rows equal +2 and the product of rows $v+1$ to $2 v$ with the first two rows equal 0 .

\begin{tabular}{|c|c|c|c|c|c|c|c|c|c|}
\hline \multirow{2}{*}{\multicolumn{2}{|c|}{$\begin{array}{c}2 \times 2 \\
\text { subsquare }\end{array}$}} & \multicolumn{8}{|c|}{ Number of Rows of Each Type Ib } \\
\hline & & $u_{1}$ & $u_{2}$ & $u_{3}$ & $u_{4}$ & $u_{5}$ & $u_{6}$ & $u_{7}$ & $u_{8}$ \\
\hline 1 & $\begin{array}{l}1 \\
1\end{array}$ & $\lambda-2$ & $k-\lambda$ & $k-\lambda$ & $v-2 k+\lambda$ & $\lambda$ & $k-\lambda$ & $k-\lambda$ & $v-2 k+\lambda$ \\
\hline- & 1 & $\lambda$ & $k-\lambda-2$ & $k-\lambda$ & $v-2 k+\lambda$ & $\lambda$ & $k-\lambda$ & $k-\lambda$ & $v-2 k+\lambda$ \\
\hline 1 & - & $\lambda$ & $k-\lambda$ & $k-\lambda-2$ & $v-2 k+\lambda$ & $\lambda$ & $k-\lambda$ & $k-\lambda$ & $v-2 k+\lambda$ \\
\hline- & - & $\lambda$ & $k-\lambda$ & $k-\lambda$ & $v-2 k+\lambda-2$ & $\lambda$ & $k-\lambda$ & $k-\lambda$ & $v-2 k+\lambda$ \\
\hline
\end{tabular}

Table 2

These reduce to three cases to test as the cases for $\begin{aligned} & - \\ & -\end{aligned}$ and $\begin{aligned} & 1 \\ & -\end{aligned}$ - give permutations, only of the terms to be evaluated.

\section{Case IIa}

A similar argument to that for Case Ia shows that using the permutations of columns one and two we only have to consider the submatrices

(i)

$$
\begin{aligned}
& 1 y-y \\
& 1 \bar{y}-\bar{y}
\end{aligned}
$$

\begin{tabular}{|c|c|c|c|c|c|c|c|c|c|}
\hline \multirow{2}{*}{\multicolumn{2}{|c|}{$\begin{array}{c}2 \times 2 \\
\text { subsquare }\end{array}$}} & \multicolumn{8}{|c|}{ Number of Rows of Each Type IIa } \\
\hline & & $u_{1}$ & $u_{2}$ & $u_{3}$ & $u_{4}$ & $u_{5}$ & $u_{6}$ & $u_{7}$ & $u_{8}$ \\
\hline $\begin{array}{l}1 \\
1\end{array}$ & $\begin{array}{l}1 \\
-\end{array}$ & $\lambda-1$ & $k-\lambda$ & $k-\lambda$ & $v-2 k+\lambda$ & $\lambda$ & $k-\lambda-1$ & $k-\lambda$ & $v-2 k+\lambda$ \\
\hline- & 1 & $\lambda$ & $k-\lambda$ & $k-\lambda-1$ & $v-2 k+\lambda$ & $\lambda$ & $k-\lambda$ & $k-\lambda$ & $v-2 k+\lambda-1$ \\
\hline $\begin{array}{l}1 \\
1\end{array}$ & $\overline{1}$ & $\lambda$ & $k-\lambda-1$ & $k-\lambda$ & $v-2 k+\lambda$ & $\lambda-1$ & $k-\lambda$ & $k-\lambda$ & $v-2 k+\lambda$ \\
\hline $\begin{array}{l}- \\
-\end{array}$ & $\overline{1}$ & $\lambda$ & $k-\lambda$ & $k-\lambda$ & $v-2 k+\lambda-1$ & $\lambda$ & $k-\lambda$ & $k-\lambda-1$ & $v-2 k+\lambda$ \\
\hline
\end{tabular}

for $y=1$ and $y=-1$. These give the results for Table 3 .

These reduce to three cases to test as the cases for $\begin{array}{lllll}- & 1 \\ - & -\end{array}$ and $\begin{array}{ccc}1 & - \\ 1 & 1\end{array}$ give permutations, only of the terms to be evaluated.

\section{Case IIb}

A similar argument to that for Case Ia shows that using the permutations of columns 1 and 2 we only have to consider the submatrices

(i)

(ii)

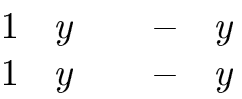

for $y=1$ and $y=-1$. These give the results for Table of IIb. 


\begin{tabular}{|c|c|c|c|c|c|c|c|c|c|}
\hline \multicolumn{2}{|c|}{$\begin{array}{c}2 \times 2 \\
\text { subsquare }\end{array}$} & \multicolumn{8}{|c|}{ Number of Rows of Each Type IIb } \\
\hline 1 & 1 & $\lambda-1$ & $k-\lambda$ & $k-\lambda$ & $v-2 k+\lambda$ & $\lambda-1$ & $k-\lambda$ & $k-\lambda$ & $v-2 k+\lambda$ \\
\hline 1 & 1 & $1-1$ & $n$ & n & $0-2 n+1$ & $x-1$ & $n$ & $n-\lambda$ & $0-2 k+\lambda$ \\
\hline- & $\begin{array}{l}1 \\
1\end{array}$ & $\lambda$ & $k-\lambda$ & $k-\lambda-1$ & $v-2 k+\lambda$ & $\lambda$ & $k-\lambda$ & $k-\lambda-1$ & $v-2 k+\lambda$ \\
\hline $\begin{array}{l}1 \\
1\end{array}$ & - & $\lambda$ & $k-\lambda-1$ & $k-\lambda$ & $v-2 k+\lambda$ & $\lambda$ & $k-\lambda-1$ & $k-\lambda$ & $v-2 k+\lambda$ \\
\hline $\begin{array}{c}- \\
-\end{array}$ & $\begin{array}{l}- \\
-\end{array}$ & $\lambda$ & $k-\lambda$ & $k-\lambda$ & $v-2 k+\lambda-1$ & $\lambda$ & $k-\lambda$ & $k-\lambda$ & $v-2 k+\lambda-1$ \\
\hline
\end{tabular}

These reduce to three cases to test as the cases for $\begin{aligned} & -1 \\ & -\end{aligned}$ and $\begin{aligned} & 1- \\ & 1\end{aligned}-$ give permutations, only of the terms to be evaluated.

\section{Case III}

We have the following $2 \times 2$ submatrices.
(i)
(ii)
$\begin{array}{llll}x & y & y & x\end{array}$
$\begin{array}{llll}x & \bar{y} & \bar{y} & x\end{array}$

One column removed comes from the columns with $2 k$ ones per column and the other from the columns with $v$ ones per column in the original design. This means the generic form of these two columns is

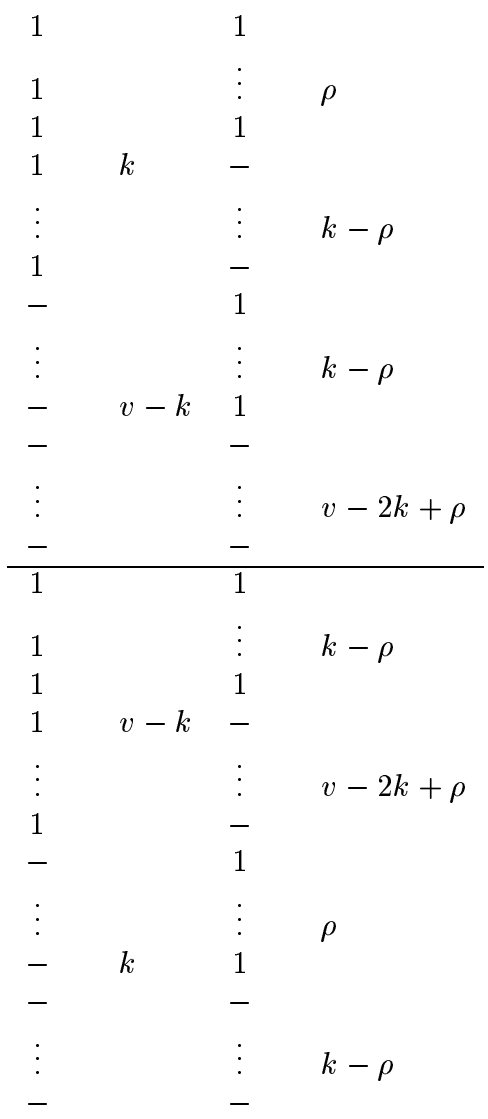

Note they have inner product zero. Also note $0 \leq \rho \leq k$. We have not proceeded to eliminate cases for $\rho$ except where $\rho-1<0$. The table lists the possible cases that arise. In the case of 


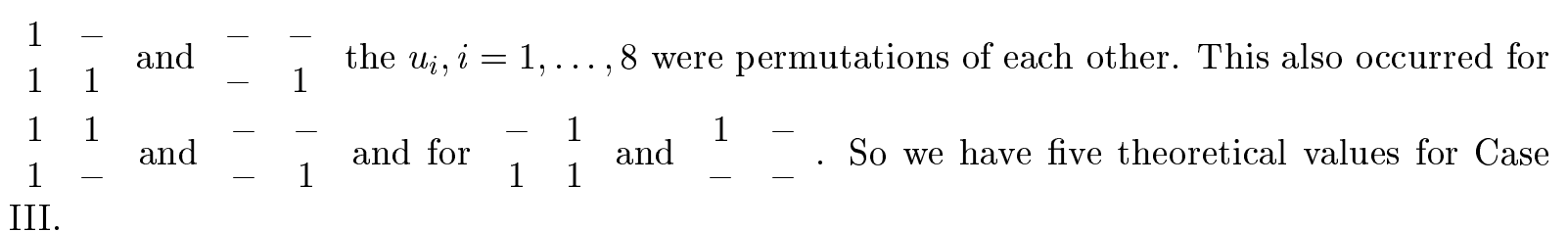

\begin{tabular}{|c|c|c|c|c|c|c|c|c|c|}
\hline \multirow{2}{*}{\multicolumn{2}{|c|}{$\begin{array}{c}2 \times 2 \\
\text { subsquare }\end{array}$}} & \multicolumn{8}{|c|}{ Number of Rows of Each Type III } \\
\hline & & $u_{1}$ & $u_{2}$ & $u_{3}$ & $u_{4}$ & $u_{5}$ & $u_{6}$ & $u_{7}$ & $u_{8}$ \\
\hline $\begin{array}{l}1 \\
1\end{array}$ & $\begin{array}{l}1 \\
-\end{array}$ & $\rho-1$ & $k-\rho$ & $k-\rho$ & $v-2 k+\rho$ & $k-\rho$ & $v-2 k+\rho-1$ & $\rho$ & $k-\rho$ \\
\hline $\begin{array}{l}1 \\
1\end{array}$ & $\overline{1}$ & $\rho$ & $k-\rho-1$ & $k-\rho$ & $v-2 k+\rho$ & $k-\rho-1$ & $v-2 k+\rho$ & $\rho$ & $k-\rho$ \\
\hline 1 & $\begin{array}{l}1 \\
1\end{array}$ & $\rho-1$ & $k-\rho$ & $k-\rho$ & $v-2 k+\rho$ & $k-\rho$ & $v-2 k+\rho$ & $\rho-1$ & $k-\rho$ \\
\hline$\overline{1}$ & $\begin{array}{l}1 \\
1\end{array}$ & $\rho$ & $k-\rho$ & $k-\rho-1$ & $v-2 k+\rho$ & $k-\rho-1$ & $v-2 k+\rho$ & $\rho$ & $k-\rho$ \\
\hline- & - & $\rho$ & $k-\rho$ & $k-\rho$ & $v-2 k+\rho-1$ & $k-\rho$ & $v-2 k+\rho-1$ & $\rho$ & $k-\rho$ \\
\hline
\end{tabular}

Lemma 2 The $(2 v-2) \times(2 v-2)$ minors of the D-optimal design of the $W B$ series are

$$
(2 s+1)(s+1) s \mathcal{T}, \quad(2 s+1) s^{2} \mathcal{T}, \quad 2 s^{2}(s+1) \mathcal{T}, \quad s\left(2 s^{2}+2 s+1\right) \mathcal{T}, \quad 2 s(s+1)^{2} \mathcal{T}, \quad 2 s^{3} \mathcal{T}
$$

where $s$ is an odd prime power, $s=2,4,6$ or 8 and $\mathcal{T}=2^{4 s^{2}+4 s-1} s^{2 s^{2}+2 s-3}(s+1)^{2 s^{2}+2 s-2}$.

Proof. Here $\lambda=\frac{1}{2} s(s-1), \quad k=s^{2}$, and $v=2 s^{2}+2 s+1$. The expressions for $u_{i}, i=1, \ldots, 8$ were calculated in each case. Maple was then used to evaluate the determinant for $D$ giving the required result. Ia and IIa give the values $2^{7} s^{2}(2 s+1)(s+1)^{3}$ and $2^{7} s^{3}(2 s+1)(s+1)^{2}$.

Cases Ib and IIb give the value zero for the determinant.

Case III give the values $2^{7} s^{3}(s+1)^{3}, 2^{7}\left(2 s^{2}+2 s+1\right)(s+1)^{2} s^{2}, 2^{8} s^{2}(s+1)^{4}$ and $2^{8} s^{4}(s+1)^{2}$. Multiplying by $\left(4 s^{2}+4 s\right)^{2 s^{2}+2 s-4}$ gives the required result.

\section{Pivot structure for $W B$ family of $D$-optimal designs}

\section{Conjecture (The growth conjecture for WB family)}

Let $A$ be an $2 v \times 2 v$ CP $D$-optimal design of WB family which is constructed from $2-\left\{2 s^{2}+\right.$ $\left.2 s+1 ; s^{2}, s^{2} ; s(s-1)\right)$ sds. Reduce $A$ by GE. Then we conjecture

(i) $g(v, A)=2 s(2 s+1)$, or $2(s+1)(2 s+1)$;

(ii) The last pivot is equal to $2 s(2 s+1)$ or $2(s+1)(2 s+1)$;

(iii) The second last pivot can take the values $2 s(s+1)=\frac{2 v-1}{2}, 2 s^{2}, 2(s+1)^{2},(s+1)(2 s+1)$, $\frac{(2 s+1) 2 s(s+1)^{2}}{\left(2 s^{2}+2 s+1\right)}, s(2 s+1), \frac{(s+1)^{2}(2 s+1)}{s}, \frac{(s)^{2}(2 s+1)}{(s+1)}$ and $\frac{(2 s+1) 2 s^{2}(s+1)}{\left(2 s^{2}+2 s+1\right)}$;

(iv) Every pivot before the last has magnitude at most $2 v$;

(v) The first four pivots are equal to 1,2,2,4;

(vi) The fifth pivot may be 2 or 3 . 
We prove (ii) and (iii) in this paper. (v) and (vi) were proved for Brouwer's $\operatorname{SBIBD}\left(2 s^{2}+2 s+\right.$ $\left.1, s^{2}, \frac{1}{2} s(s-1)\right)$ in [11] and we also show they hold for the WB family.

Theorem 1 Let $A$ be the $2 v \times 2 v$ D-optimal design of the WB family. Reduce $A$ by GECP. Then the last pivots are $2 s(2 s+1)$ and $2(s+1)(2 s+1)$. The second last pivots are $2 s(s+1)$, $2 s^{2}, 2(s+1)^{2},(s+1)(2 s+1), \frac{(2 s+1) 2 s(s+1)^{2}}{\left(2 s^{2}+2 s+1\right)}, s(2 s+1), \frac{(s+1)^{2}(2 s+1)}{s}, \frac{(s)^{2}(2 s+1)}{(s+1)}$ and $\frac{(2 s+1) 2 s^{2}(s+1)}{\left(2 s^{2}+2 s+1\right)}$

Proof. ¿From (4) and lemma's 1 and 2 we have for the $D$-optimal design made using $2-\left\{2 s^{2}+\right.$ $\left.2 s+1 ; s^{2}, s^{2} ; s(s-1)\right\}$ sds, the results in Table 6 where the first row gives the values of $M_{2 v-1}$, the first column gives the values of $M_{2 v-2}$ and the entries are $p_{2 v}=\frac{M_{2 v-1}}{M_{2 v-2}}$.

\begin{tabular}{c|c|c|}
$M_{2 v-1}$ & $2 s^{2}(s+1)^{2}(2 s+1) \mathcal{T}$ & $2 s^{3}(s+1)(2 s+1) \mathcal{T}$ \\
\hline$M_{2 v-2}$ & & \\
$(2 s+1)(s+1) \mathcal{T}$ & $2 s(s+1)^{*}$ & $2 s^{2}$ \\
$(2 s+1) s^{2} \mathcal{T}$ & $2(s+1)^{2}$ & $2 s(s+1)$ \\
$2 s^{2}(s+1) \mathcal{T}$ & $(s+1)(2 s+1)$ & $s(2 s+1)$ \\
$s\left(2 s^{2}+2 s+1\right) \mathcal{T}$ & $\frac{2 s(s+1)^{2}(2 s+1)^{*}}{\left(2 s^{2}+2 s+1\right)}$ & $\frac{2 s^{2}(s+1)(2 s+1)}{\left(2 s^{2}+2 s+1\right)}$ \\
$2 s(s+1)^{2} \mathcal{T}$ & $s(2 s+1)^{*}$ & $\frac{s^{2}(2 s+1)}{(s+1)}$ \\
$2 s^{3} \mathcal{T}$ & $\frac{(s+1)^{2}(2 s+1)}{s}$ & $(s+1)(2 s+1)$ \\
\hline \multicolumn{2}{|r}{ Table 6}
\end{tabular}

The entries marked $*$ are these obtained in experiments.

We give some values for the family WB.

\begin{tabular}{c|c|cc|cccc|}
$2 v$ & $s$ & \multicolumn{5}{|c}{$p_{2 v}$} & $p_{2 v-1}$ \\
& & $2 s(2 s+1)$ & $2(s+1)(2 s+1)$ & $2 s(s+1)$ & $2(s+1)^{2}$ & $2 s^{2}$ & $(s+1)(2 s+1)$ \\
\hline 26 & 2 & 20 & 30 & 12 & 18 & 8 & 15 \\
50 & 3 & 42 & 56 & 24 & 32 & 18 & 28 \\
82 & 4 & 72 & 90 & 40 & 50 & 32 & 45
\end{tabular}

\begin{tabular}{c|c|ccccc|}
$2 v$ & $s$ & \multicolumn{5}{|c}{$p_{2 v-1}$} \\
& & $s(2 s+1)$ & $\frac{2 s(s+1)^{2}(2 s+1)}{\left(2 s^{2}+2 s+1\right)}$ & $\frac{2 s^{2}(s+1)(2 s+1)}{\left(2 s^{2}+2 s+1\right)}$ & $\frac{s^{2}(2 s+1)}{(s+1)}$ & $\frac{(s+1)^{2}(2 s+1)}{s}$ \\
\hline 26 & 2 & 10 & $\frac{180}{13}$ & $\frac{120}{13}$ & $\frac{20}{3}$ & $\frac{45}{2}$ \\
50 & 3 & 21 & $\frac{672}{25}$ & $\frac{704}{25}$ & $\frac{63}{4}$ & $\frac{112}{3}$ \\
82 & 4 & 36 & $\frac{1800}{41}$ & $\frac{1440}{41}$ & $\frac{144}{5}$ & $\frac{225}{4}$
\end{tabular}

Remark 1 We experimented with $2 v=26$. The theoretical values for $M_{2 v-1}$ are $2^{35} \cdot 5 \cdot 3^{12}$ and $2^{36} \cdot 5 \cdot 3^{11}$. In our calculations we found always $p_{2 v}=20$ and $p_{2 v-1}=12$ or 10 or $\frac{180}{13}$. This leaves as an open problem the existence of a $26 \times 26$ matrix having growth equal to 30 . 
The next result is easy to prove using a counting argument and noting the inner product of every pair of rows is +1 to see that the design always contains a $4 \times 4$ Hadamard matrix.

Proposition 1 Let $A$ be the $2 v \times 2 v(1,-1)$ incidence matrix of an SBIBD of the WB family. Reduce $A$ by GECP then the magnitudes of the first four pivots are 1,2,2 and 4; the magnitude of $\left|a_{55}^{(4)}\right|$ is 2 or 3 .

Proof: Since the design always contains a $4 \times 4$ Hadamard matrix, this can be moved to be the $4 \times 4$ principal minor without changing the $\mathrm{CP}$ property. Thus the first four pivots will be $1,2,2$ and 4 [4]. Because every entry in $A^{(3)}$ is of magnitude 0,2 or 4 , pivoting on $a_{44}^{(3)}$ will only involve adding \pm 1 or $\pm 1 / 2$ times the fourth row of $A^{(3)}$ to the rows below, and this will create only integer entries in $A^{(4)}$. It is known, see Payne [14], that if $v \equiv 1(\bmod ) 4, \quad v \neq 1, \quad d_{v} \leq(v-1)^{\frac{v-1}{2}} \sqrt{2 v-1}$ and equality can hold only if $v=2 s^{2}+2 s+1, \quad s=1,2,3, \ldots$ Thus $\left|a_{55}^{(4)}\right|$ must be an integer satisfying the relation

$$
A(12345)=16\left|a_{55}^{(4)}\right| \leq 4^{4 / 2} \sqrt{10-1} \Rightarrow\left|a_{55}^{(4)}\right| \leq 3 .
$$

where $A(12345)$ denotes the determinant of the $5 \times 5$ principal submatrix of $A$. Thus $\left|a_{55}^{(4)}\right|$ must be 1,2 or 3 . To see that it cannot be 1 is to show that one could not have

$$
A^{(4)}\left[\begin{array}{lllll}
1 & & & & \\
& 2 & & & \\
& & 2 & & \\
& & & 4 & \\
& & & & B
\end{array}\right]
$$

where every entry of $B$ is zero or \pm 1 ; for, if that were true, then $B$ would be a normalized $(v-4) \times(v-4)$ matrix, and so

$$
|\operatorname{det} B| \leq(v-4)^{\frac{v-4}{2}} .
$$

But $|\operatorname{det} B|=\frac{(v-1)^{\frac{v-1}{2}} \sqrt{2 v-1}}{16}$ and it is easily checked that these cannot both hold when $v>4$.

By detecting the pivot structure of WB, Table 8 was computed. The first nine pivots and the last two are presented. All the other intermediate pivots take a variety of values. At least 837 different pivot structures were detected for $2 v=26$ and 500 for $2 v=50$.

\begin{tabular}{|c|c|c|c|}
\hline$s$ & $v$ & growth & Pivot Pattern \\
\hline 2 & 26 & 20 & $\left(1,2,2,4,3, \frac{10}{3}, \frac{18}{5}, 4, \frac{18}{4}, \ldots, 12,20\right)$ \\
\hline 2 & 26 & 20 & $\left(1,2,2,4,3, \frac{10}{3}, \frac{18}{5}, 4, \frac{44}{9}, \ldots, \frac{180}{13}, 20\right)$ \\
\hline 2 & 26 & 20 & $\left(1,2,2,4,3, \frac{10}{3}, \frac{18}{5}, 4,4, \ldots, 10,20\right)$ \\
\hline 3 & 50 & 42 & $\left(1,2,2,4,3, \frac{10}{3}, \frac{18}{5}, 4,5, \ldots, 24,42\right)$ \\
\hline 3 & 50 & 42 & $\left(1,2,2,4,3, \frac{10}{3}, \frac{18}{5}, 4,4, \ldots, 21,42\right)$ \\
\hline 4 & 82 & 72 & $\left(1,2,2,4,3, \frac{10}{3}, \frac{18}{5}, 4,4, \ldots, 40,72\right)$ \\
\hline 5 & 122 & 110 & $\left(1,2,2,4,3, \frac{10}{3}, \frac{18}{5}, 4,4, \ldots, 60,110\right)$ \\
\hline
\end{tabular}

Table 8: Growth Factors and Pivots Patterns for small CP WB designs 


\section{References}

[1] T. Beth, D. Jungnickel and H. Lenz, Design Theory, Cambridge University Press, Cambridge, 1985.

[2] W.G. Bridges, M. Hall Jr. and J.L. Hayden, Codes and designs, J. Combin. Theory Ser. A, 31 (1981), 155-174.

[3] A.E. Brouwer, An infinite series of symmetric designs, Math. Centrum Amsterdam Report ZW 202/83, 1983.

[4] J. Day and B. Peterson, Growth in Gaussian elimination, Amer. Math. Monthly, 95 (1988), 489-513.

[5] D.Z. Djokovic, Some new D-optimal designs, Australas. J. Combin., 15 (1997), 221-231.

[6] H. Ehlich, Determinantenabschätzungen für binäre matrizen, Math. Z., 83 (1964), 123-132.

[7] S. Georgiou and C. Koukouvinos, On multipliers of supplementary difference sets and $D$ optimal designs for $n \equiv 2 \operatorname{mod4}$, Utilitas Math., 56 (1999), 127-136.

[8] M. Gysin, New D-optimal designs via cyclotomy and generalised cyclotomy, Australas. J. Combin., 15 (1997), 247-255.

[9] N. J. Higham, Accuracy and Stability of Numerical Algorithms, SIAM, Philadelphia, 1996.

[10] C. Koukouvinos, S. Kounias, and J. Seberry, Supplementary difference sets and optimal designs, Discrete Math., 88 (1991), 49-58.

[11] C. Koukouvinos, M. Mitrouli and J. Seberry, Values of minors of $(1,-1)$ incidence matrices of SBIBDs and their application to the growth problem, Designs, Codes and Cryptography, (to appear).

[12] C. Koukouvinos, M. Mitrouli and J. Seberry, Growth in Gaussian elimination for weighing matrices $W(n, n-1)$, Linear Algebra and its Applications, 306 (2000), 189-202.

[13] C. Koukouvinos, J. Seberry, A.L. Whiteman and M.Y.Xia, Optimal designs, supplementary difference sets and multipliers, J. Statist. Plann. Inference, 62 (1997), 81-90.

[14] S. E. Payne, On maximizing $\operatorname{det}\left(A A^{T}\right)$, Discrete Math., 10 (1974), 145-158.

[15] A.P. Street and D.J. Street, Combinatorics of Experimental Design, Oxford University Press, Oxford, 1987.

[16] T. van Trung, The existence of symmetric block designs with parameters $(41,16,6)$ and (66, 26, 10), J. Combin. Theory Ser. A, 33 (1982), 201-204.

[17] T. van Trung, Symmetric designs, in CRC Handbook of Combinatorial Designs, C.J. Colbourn and J.H. Dinitz (Eds.), CRC Press, Boca Raton, (1996), 75-87.

[18] A.L. Whiteman, A family of D-optimal designs, Ars Combinatoria, 30 (1990), 23-26.

[19] J. H. Wilkinson, The Algebraic Eigenvalue Problem, Oxford University Press, London, 1988. 


\section{Appendix A: The Determinant Simplification Theorem}

We use the notation

$$
C C^{T}=\left(k-a_{i i}\right) I_{b_{1}, b_{2}, \cdots, b_{z}}+a_{i j} J_{b_{1}, b_{2}, \cdots, b_{z}}
$$

for a matrix of blocks with integer multiples. For example the matrix

$$
\begin{aligned}
& C C^{T}=\left(k-a_{i i}\right) I_{u, v, w, x}+a_{i j} J_{u, v, w, x} \\
& \text { where }\left(a_{i j}\right)=\left[\begin{array}{llll}
a & b & c & d \\
b & a & e & f \\
c & e & a & g \\
d & f & g & a
\end{array}\right] \text { is the }(u+v+w+x) \times(u+v+w+x) \text { matrix }
\end{aligned}
$$

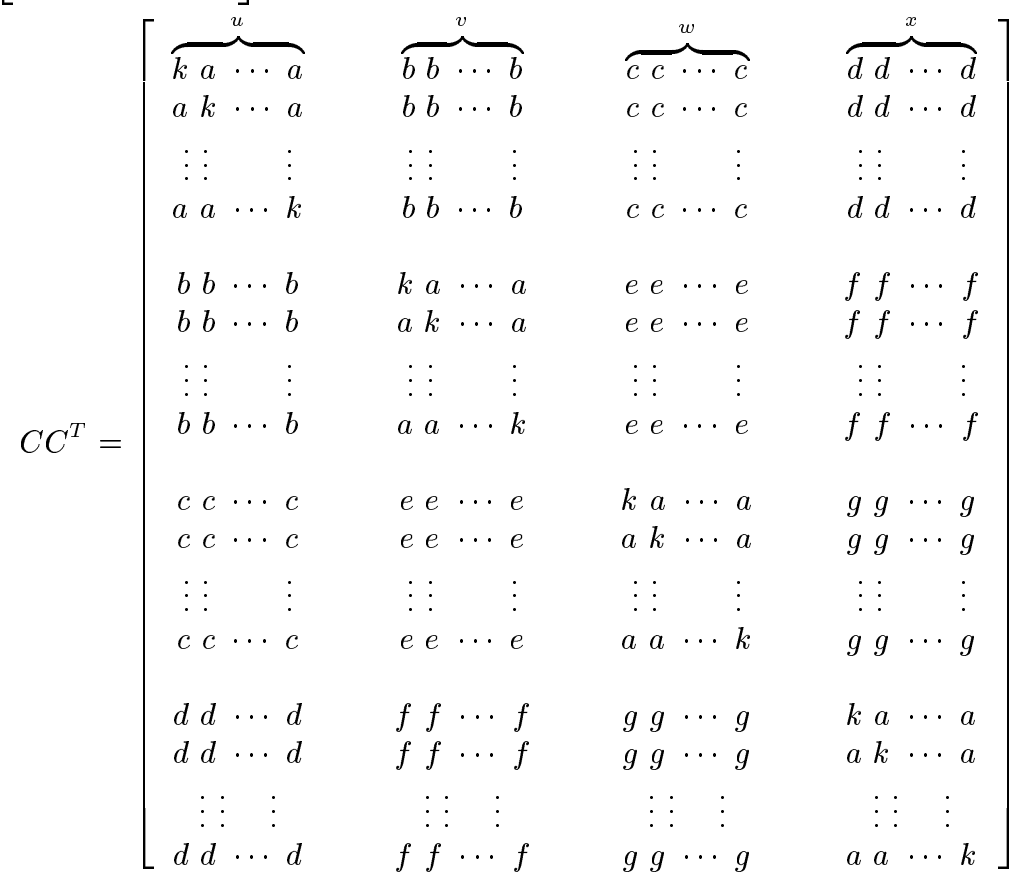

We now give a theorem proved similarly to the proof for finding the determinant of an SBIBD in [15, Theorem 3,p.32].

Theorem 2 (Determinant Simplification Theorem) Let

$$
C C^{T}=\left(k-a_{i i}\right) I_{b_{1}, b_{2}, \cdots, b_{z}}+a_{i j} J_{b_{1}, b_{2}, \cdots, b_{z}}
$$

then

$$
\operatorname{det} C C^{T}=\Pi_{i=1}^{z}\left(k-a_{i i}\right)^{b_{i}-1} \operatorname{det} D
$$

where

$$
D=\left[\begin{array}{ccccc}
k+\left(b_{1}-1\right) a_{11} & b_{2} a_{12} & b_{3} a_{13} & \cdots & b_{z} a_{1 z} \\
b_{1} a_{21} & k+\left(b_{2}-1\right) a_{22} & b_{3} a_{23} & \cdots & b_{z} a_{2 z} \\
\vdots & \vdots & \vdots & & \vdots \\
b_{1} a_{z 1} & b_{2} a_{z 2} & b_{3} a_{z 2} & \cdots & k+\left(b_{z}-1\right) a_{z z}
\end{array}\right]
$$


Corollary 1 Suppose $C$ is the matrix of order $(u+v+w+x) \times(u+v+w+x)$, where $n=u+v+w+x$, for which $C C^{T}$ is given above, satisfying $C C^{T}=\left(k-a_{i i}\right) I_{u, v, w, x}+a_{i j} J_{u, v, w, x}$. Then

$$
\operatorname{det} C C^{T}=(k-a)^{n-4} \operatorname{det} D
$$

where

$$
D=\left[\begin{array}{cccc}
k+(u-1) a & v b & w c & x d \\
u b & k+(v-1) a & w e & x f \\
u c & v e & k+(w-1) a & x g \\
u d & v f & w g & k+(x-1) a
\end{array}\right]
$$

